\title{
Cisplatin, Fluorouracil, and Docetaxel in Unresectable Head and Neck Cancer
}

\author{
Jan B. Vermorken, M.D., Ph.D., Eva Remenar, M.D., Carla van Herpen, M.D., Ph.D., \\ Thierry Gorlia, M.Sc., Ricard Mesia, M.D., Marian Degardin, M.D., \\ John S. Stewart, M.D., Svetislav Jelic, M.D., Jan Betka, M.D., \\ Joachim H. Preiss, M.D., Ph.D., Danielle van den Weyngaert, M.D., \\ Ahmad Awada, M.D., Ph.D., Didier Cupissol, M.D., Heinz R. Kienzer, M.D., \\ Augustin Rey, M.D., Isabelle Desaunois, M.Sc., Jacques Bernier, M.D., Ph.D., \\ and Jean-Louis Lefebvre, M.D., for the EORTC 24971/TAX 323 Study Group*
}

\section{A BSTRACT}

\section{BACKGROUND}

Phase 2 studies suggest that the standard regimen of cisplatin and fluorouracil (PF) plus docetaxel (TPF) improves outcomes in squamous-cell carcinoma of the head and neck. We compared TPF with PF as induction chemotherapy in patients with locoregionally advanced, unresectable disease.

\section{METHODS}

We randomly assigned eligible patients between the ages of 18 and 70 years who had stage III or stage IV disease and no distant metastases to receive either TPF (docetaxel and cisplatin, day 1; fluorouracil by continuous infusion, days 1 to 5) or PF every 3 weeks for four cycles. Patients without progression of disease received radiotherapy within 4 to 7 weeks after completing chemotherapy. The primary end point was progression-free survival.

\section{RESULTS}

A total of 358 patients underwent randomization, with 177 assigned to the TPF group and 181 to the PF group. At a median follow-up of 32.5 months, the median progression-free survival was 11.0 months in the TPF group and 8.2 months in the PF group (hazard ratio for disease progression or death in the TPF group, 0.72; $\mathrm{P}=0.007)$. Treatment with TPF resulted in a reduction in the risk of death of $27 \%$ $(\mathrm{P}=0.02)$, with a median overall survival of 18.8 months, as compared with 14.5 months in the PF group. There were more grade 3 or 4 events of leukopenia and neutropenia in the TPF group and more grade 3 or 4 events of thrombocytopenia, nausea, vomiting, stomatitis, and hearing loss in the PF group. The rates of death from toxic effects were $2.3 \%$ in the TPF group and $5.5 \%$ in the PF group.

\section{CONCLUSIONS}

As compared with the standard regimen of cisplatin and fluorouracil, induction chemotherapy with the addition of docetaxel significantly improved progressionfree and overall survival in patients with unresectable squamous-cell carcinoma of the head and neck. (ClinicalTrials.gov number, NCT00003888.)
From Universitair Ziekenhuis Antwerpen, Edegem, Belgium (J.B.V.); the National Institute of Oncology, Budapest, Hungary (E.R.); Universitair Medisch Centrum Nijmegen, Nijmegen, the Netherlands (C.H.); EORTC Data Center, Brussels (T.G., I.D.); Institut Catala d'Oncologia, Barcelona (R.M.); Centre Oscar Lambret, Lille, France (M.D., J.-L.L.); Charing Cross Hospital, London (J.S.S.); the Institute of Oncology and Radiology, Belgrade, Serbia (S.J.); University Hospital Motol, Prague, Czech Republic (J. Betka); Caritasklinik St. Theresia, Saarbrucken, Germany (J.H.P.); Ziekenhuis Netwerk Antwerpen Middelheim, Antwerp, Belgium (D.W.); Institut Jules Bordet, Brussels (A.A.); Centre Régional de Lutte contre le Cancer Val d'Aurelle, Montpellier, France (D.C.); Kaiser Franz Josef Spital, Vienna (H.R.K.); SanofiAventis Global Oncology, Paris (A.R.); and Clinique de Genolier, Genolier, Switzerland (J. Bernier). Address reprint requests to Dr. Vermorken at the Department of Medical Oncology, Universitair Ziekenhuis Antwerpen, Wilrijkstraat 10, 2650 Edegem, Belgium, or at jan.b.vermorken@uza.be.

*Investigators for the European Organization for Research and Treatment of Cancer (EORTC) are listed in the Supplementary Appendix, available with the full text of this article at www.nejm.org.

N Engl J Med 2007;357:1695-704.

Copyright (c) 2007 Massachusetts Medical Society. 
$\mathrm{H}$ EAD AND NECK CANCER, MOSTLY OF squamous-cell origin, ranks sixth among the most common cancers, accounting for approximately $6 \%$ of all cases of cancer. Each year, more than 500,000 new cases are diagnosed worldwide. ${ }^{1}$ Approximately $60 \%$ of patients present with advanced disease (stages III and IV), for which the prognosis is poor. ${ }^{2}$ For many years, radiotherapy has been the treatment of choice for unresectable disease, ${ }^{3}$ resulting in 5 -year rates of survival of less than $20 \% .^{4,5}$

Concurrent chemoradiotherapy has now largely replaced radiotherapy alone in the treatment of unresectable squamous-cell carcinoma of the head and neck, 3,6 but induction chemotherapy has also been associated with a survival benefit and could be a valuable treatment option. ${ }^{5,7,8} \mathrm{~A}$ beneficial effect of induction chemotherapy has been observed only with the Wayne State University regimen of cisplatin (at a dose of $100 \mathrm{mg}$ per square meter of body-surface area) plus infusional fluorouracil (at a dose of $1000 \mathrm{mg}$ per square meter per day for 5 days), a treatment that has emerged as the most active combination ${ }^{9}$ and that outperformed other induction regimens in a large meta-analysis. ${ }^{10}$ For this reason, this regimen can be considered a good comparator in randomized trials of new induction regimens of chemotherapy. The best number of treatment cycles is uncertain, but complete response rates increased with each successive cycle up to cycle 4 in the study by Paccagnella et al. ${ }^{5}$

Among agents introduced in the 1990s, the taxanes have shown great promise for the treatment of squamous-cell carcinoma of the head and neck. ${ }^{11}$ Phase 2 studies indicate that docetaxel plus cisplatin and fluorouracil (TPF) might be more efficacious than the classic regimen of cisplatin plus fluorouracil (PF). ${ }^{12}$ The Head and Neck Cancer Group of the European Organization for Research and Treatment of Cancer (EORTC) conducted a phase 3 trial, called the EORTC 24971/TAX 323 study, comparing TPF with PF as induction chemotherapy before radiotherapy in patients with locoregionally advanced, unresectable squamous-cell carcinoma of the head and neck.

\section{METHODS}

\section{PATIENTS}

We conducted an open-label, randomized, stratified phase 3 study at 37 institutions in 15 Euro- pean countries. The protocol was approved by the ethics committee or institutional review board at each participating center, and all patients provided written informed consent.

Eligible patients were between the ages of 18 and 70 years and had squamous-cell carcinoma of the head and neck that had been diagnosed by histologic or cytologic analysis. All patients had previously untreated and locoregionally advanced disease with at least one lesion that was measurable either unidimensionally or bidimensionally, a tumor-node-metastasis (TNM) stage of III or IV without metastases, a World Health Organization (WHO) performance status of 1 or less, and adequate hematologic, renal, and hepatic function. Tumors had to be considered unresectable by a multidisciplinary team. Patients with tumors of the nasopharynx and the nasal and paranasal cavities were excluded.

Evaluations were conducted within 7 days before randomization and included history taking, physical examination, hematologic and biochemical analysis, electrocardiography, magnetic resonance imaging or computed tomography (CT) of the head and neck, and chest radiography. Abdominal ultrasonography or CT and bone scanning were performed when indicated.

\section{STUDY DESIGN}

Patients were randomly assigned to study groups after verification of eligibility at the EORTC data center. Randomization was balanced according to the primary tumor site (oral cavity, oropharynx, hypopharynx, or larynx) and the center with the use of a variance-minimization method. ${ }^{13}$

Patients were randomly assigned to receive either TPF or PF. The TPF regimen consisted of docetaxel at a dose of $75 \mathrm{mg}$ per square meter, administered as a 1-hour infusion on day 1, followed by cisplatin at a dose of $75 \mathrm{mg}$ per square meter, administered as a 1-hour infusion on day 1 , and fluorouracil at a dose of $750 \mathrm{mg}$ per square meter per day, administered by continuous infusion on days 1 to 5 . The PF regimen consisted of cisplatin at a dose of $100 \mathrm{mg}$ per square meter, administered as a 1-hour infusion on day 1, followed by fluorouracil at a dose of $1000 \mathrm{mg}$ per square meter per day, administered by continuous infusion on days 1 to 5 . Treatment was administered every 3 weeks (defined as one cycle) for up to four cycles, unless progressive disease, 
unacceptable toxic effects, or withdrawal from the study occurred earlier. During chemotherapy, patients were monitored clinically and with laboratory tests on day 1 of each cycle before treatment. Imaging of tumors was performed at the end of cycles 2 and 4.

Patients who did not have progressive disease and who had adequate bone marrow function (neutrophil count, $\geq 2.0 \times 10^{3}$ cells per cubic millimeter; platelet count, $\geq 100 \times 10^{3}$ cells per cubic millimeter; and hemoglobin, $\geq 10$ g per deciliter), complete resolution of mucositis for at least 1 week, and healing from any dental procedures underwent radiotherapy within 4 to 7 weeks after the completion of chemotherapy. Radiation was delivered during a 7-week period with the use of either conventional fractionation (total dose, 66 to $70 \mathrm{~Gy}$ ) or accelerated or hyperfractionated regimens (total maximum dose of $70 \mathrm{~Gy}$ for the accelerated regimen and 74 Gy for the hyperfractionated regimen). Each center made a commitment before the study began to use one of these methods. Neck dissection was considered for all patients before radiotherapy and 3 months after the completion of radiotherapy.

The primary end point, progression-free survival, was defined as the time from randomization to progression, relapse, or death, whichever occurred first. If progression, relapse, or death did not occur before the cutoff date, data were censored at the time of the last valid assessment before the cutoff date. Secondary end points were overall survival, best overall response rate after induction chemotherapy and after radiation therapy, duration of response, time to treatment failure, toxic effects, and health-related quality of life, as reported previously. ${ }^{14}$

The Head and Neck Cancer Group of the EORTC conceived and developed the trial, which was sponsored by Sanofi-Aventis. The company provided docetaxel and was involved in the trial design but not in the final analysis. All data were collected and analyzed by the academic authors. Dr. Vermorken, who vouches for the completeness and accuracy of the data, wrote the article with critical review by all the other authors. The article was also reviewed by industry representatives, but its final content was determined by the academic investigators.
CONCOMITANT TREATMENT AND DOSE MODIFICATIONS

All patients received adequate antiemetic medications during chemotherapy. Dexamethasone (at a dose of $8 \mathrm{mg}$ or the equivalent) was given orally for a total of six doses, starting the night before the administration of chemotherapy, to prevent hypersensitivity reactions and to reduce docetaxelrelated skin toxic effects and fluid retention. Prophylactic antibiotics (ciprofloxacin, at a dose of $500 \mathrm{mg}$ given orally twice daily, or an alternative agent) were given to the TPF group only from days 5 to 15 of each cycle. Prophylactic granulocyte colony-stimulating factor was permitted only if a patient had febrile neutropenia or infection, a delay in recovery of the absolute neutrophil count at day 28 , or grade 4 neutropenia persisting for 7 days or more.

A cycle could be delayed for up to 2 weeks to allow for a reduction in the severity of toxic events of grade 3 or more to a severity of grade 1 or less (with the exception of alopecia, fatigue, malaise, and nail changes). Delays beyond 2 weeks required discontinuation of chemotherapy. Reductions in the dose of docetaxel were planned for grade 4 neutropenia and its complications, skin reactions, elevated bilirubin levels, and impaired liver function. Modifications in the dose of cisplatin were made for peripheral sensory and motor neurotoxicity, ototoxicity, or nephrotoxicity; patients with neurotoxicity or ototoxicity of grade 3 or more were withdrawn from the study. Modifications in the dose of fluorouracil were made for mucositis and diarrhea; patients with toxic effects of grade 4 were withdrawn from the study.

\section{ASSESSMENTS}

\section{Efficacy}

Tumor responses were evaluated with the use of WHO criteria, which were modified to account for the fact that radiotherapy started as soon as possible after the end of chemotherapy. Some responses were not reevaluated 4 weeks after the assessment of the initial response.

\section{Safety}

Adverse events were graded according to the expanded common toxicity criteria of the Clinical 
Trials Group of the National Cancer Institute of Canada, which were adopted in 1994 for the assessment of morbidity associated with chemotherapy and radiation. Late adverse events associated with radiotherapy were graded according to the criteria of the EORTC's Radiation Therapy Oncology Group. Laboratory safety data were assessed before the administration of chemotherapy and on certain days during and after such treatments, as described in the protocol.

\section{STATISTICAL ANALYSIS}

The trial had a power of $85 \%$ to detect a $50 \%$ increase in median progression-free survival (15 months in the TPF group and 10 months in the $\mathrm{PF}$ group) for a hazard ratio of 0.67 . On the basis of a 24-month accrual period with a 12-month follow-up, an estimated 260 events were required from a total accrual of 330 patients (165 per treatment group). On the basis of an anticipated loss to follow-up of 5 to $10 \%$, a total of 358 patients were enrolled. With this number, the trial had a power of $90 \%$ to detect an improvement of 15 percentage points in the 1-year survival rate $(85 \%$ in the TPF group and $70 \%$ in the PF group).

A two-sided level of significance of 0.05 was applied to all tests. Time-to-event data were described with the use of Kaplan-Meier curves. Confidence intervals were calculated for median progression-free survival and overall survival. ${ }^{15}$ Time-to-event intervals were compared between groups with the multivariate Cox proportionalhazards model (primary analysis) and the logrank test. In the multivariate analysis, the following items were included in the model for each analysis: type of treatment, location of primary tumor (oral cavity, oropharynx, or hypopharynx), tumor stage, regional-node stage, and WHO score for performance status. An unadjusted chisquare test was used to compare response variables. Fisher's exact test was used to compare other binary and categorical variables. Continuous measurements and scores were compared with the Wilcoxon test.

All efficacy analyses were conducted in the intention-to-treat population. Patients could be evaluated for a response (according to modified WHO criteria) if all baseline lesions were assessed at least once after the second chemotherapy cycle by means of the same method used at baseline.
The safety population included only patients who received their randomly assigned treatment.

All patients were followed until death. Patients with progressive disease at any time were referred to the radiation oncologist according to the institution's policy and were followed for survival only.

\section{RESULTS}

\section{PATIENTS}

Between April 1999 and March 2002, a total of 358 patients were randomly assigned to study groups, with 181 assigned to receive PF and 177 assigned to receive TPF. The two treatment groups were balanced according to baseline demographic and clinical characteristics (Table 1).

\section{EXPOSURE TO STUDY TREATMENT}

The numbers of patients who were enrolled and who completed the study are shown in Figure 1. Most patients (75.7\% in the TPF group and $65.7 \%$ in the PF group) completed the chemotherapy as defined by the protocol. Treatment groups were similar with respect to the number of chemotherapy cycles and duration of treatment (Table 1 of the Supplementary Appendix, available with the full text of this article at www.nejm.org). Protocoldefined chemotherapy doses were administered in $95 \%$ of cycles in the TPF group and in $91 \%$ of cycles in the PF group. Before the scheduled completion of the study, 38 patients in the TPF group and 60 in the PF group discontinued chemotherapy $(\mathrm{P}=0.02)$. The most frequent reasons for discontinuation were progressive disease $(7.9 \%$ in the TPF group and $7.2 \%$ in the PF group), adverse events $(6.2 \%$ in the TPF group and $11.6 \%$ in the PF group), and death (3.4\% in the TPF group and $6.6 \%$ in the PF group).

Dose reductions were made mostly because of nonhematologic toxic effects. Dose delays were more common in the PF group (Table 1 of the Supplementary Appendix). The most frequent reasons for dose delays were hematologic toxic effects in the PF group $(64.8 \%$, vs. $10.9 \%$ in the TPF group; $\mathrm{P}<0.001$ ) and other reasons (mainly administrative) in the TPF group $(84.8 \%$, vs. $29.5 \%$ in the PF group; $\mathrm{P}<0.001)$. The majority of patients received conventional radiotherapy, but four investigators delivered more than one type of radiotherapy. Only eight patients in the TPF group 


\begin{tabular}{|c|c|c|c|c|}
\hline Characteristic & $\begin{array}{c}P F \\
(N=181)\end{array}$ & $\begin{array}{c}\text { TPF } \\
(\mathrm{N}=177)\end{array}$ & $\begin{array}{c}\text { Total } \\
(\mathrm{N}=358)\end{array}$ & P Value \\
\hline Male sex — no. (\%) & $162(89.5)$ & $159(89.8)$ & $321(89.7)$ & 0.87 \\
\hline Age $-y r$ & & & & 0.77 \\
\hline Median & 53 & 53 & 53 & \\
\hline Range & $30-71$ & $31-70$ & $30-71$ & \\
\hline \multicolumn{5}{|l|}{ Age - no. (\%) } \\
\hline$<35 \mathrm{yr}$ & $4(2.2)$ & $2(1.1)$ & $6(1.7)$ & \\
\hline $35-49 \mathrm{yr}$ & $58(32.0)$ & $53(29.9)$ & $111(31.0)$ & \\
\hline $50-64 \mathrm{yr}$ & $101(55.8)$ & $104(58.8)$ & $205(57.3)$ & \\
\hline $65-75 \mathrm{yr}$ & $18(9.9)$ & $18(10.2)$ & $36(10.1)$ & \\
\hline WHO performance status — no. $(\%) \dagger$ & & & & 0.96 \\
\hline 0 & $91(50.3)$ & $90(50.8)$ & $181(50.6)$ & \\
\hline 1 & $90(49.7)$ & $86(48.6)$ & $176(49.2)$ & \\
\hline 2 & 0 & $1(0.6)$ & $1(0.3)$ & \\
\hline \multicolumn{5}{|l|}{ Cancer site - no. (\%) } \\
\hline Hypopharynx & $52(28.7)$ & $53(29.9)$ & $105(29.3)$ & 0.99 \\
\hline Larynx & $13(7.2)$ & $12(6.8)$ & $25(7.0)$ & \\
\hline Oral cavity & $32(17.7)$ & $31(17.5)$ & $63(17.6)$ & \\
\hline Oropharynx & $84(46.4)$ & $81(45.8)$ & $165(46.1)$ & \\
\hline Tumor status — no. (\%) & & & & 0.50 \\
\hline $\mathrm{Tl}$ & $1(0.6)$ & $3(1.7)$ & $4(1.1)$ & \\
\hline $\mathrm{T} 2$ & $15(8.3)$ & $10(5.6)$ & $25(7.0)$ & \\
\hline $\mathrm{T} 3$ & 36 (19.9) & $41(23.2)$ & $77(21.5)$ & \\
\hline $\mathrm{T} 4$ & $129(71.3)$ & $123(69.5)$ & $252(70.4)$ & \\
\hline Node status - no. (\%) & & & & 0.09 \\
\hline NO & $26(14.4)$ & $16(9.0)$ & $42(11.7)$ & \\
\hline $\mathrm{N} 1$ & $29(16.0)$ & $27(15.3)$ & $56(15.6)$ & \\
\hline N2 & $103(56.9)$ & $102(57.6)$ & $205(57.3)$ & \\
\hline N3 & $20(11.0)$ & $32(18.1)$ & $52(14.5)$ & \\
\hline Unknown & $3(1.7)$ & 0 & $3(0.8)$ & \\
\hline Disease sites in head and neck — no. (\%) & & & & 0.77 \\
\hline 1 & $25(13.8)$ & $19(10.7)$ & $44(12.3)$ & \\
\hline 2 & $140(77.3)$ & $150(84.7)$ & $290(81.0)$ & \\
\hline$\geq 3$ & $13(7.2)$ & $7(4.0)$ & $20(5.6)$ & \\
\hline No tumor assessment at baseline - no. (\%) & $3(1.7)$ & $1(0.6)$ & $4(1.1)$ & \\
\hline
\end{tabular}

* PF denotes cisplatin-fluorouracil, TPF docetaxel-cisplatin-fluorouracil, and WHO World Health Organization. Percentages may not total 100 because of rounding.

$\dagger$ A performance status of 0 denotes asymptomatic, 1 symptomatic and fully ambulatory, and 2 symptomatic and in bed less than $50 \%$ of the day. 


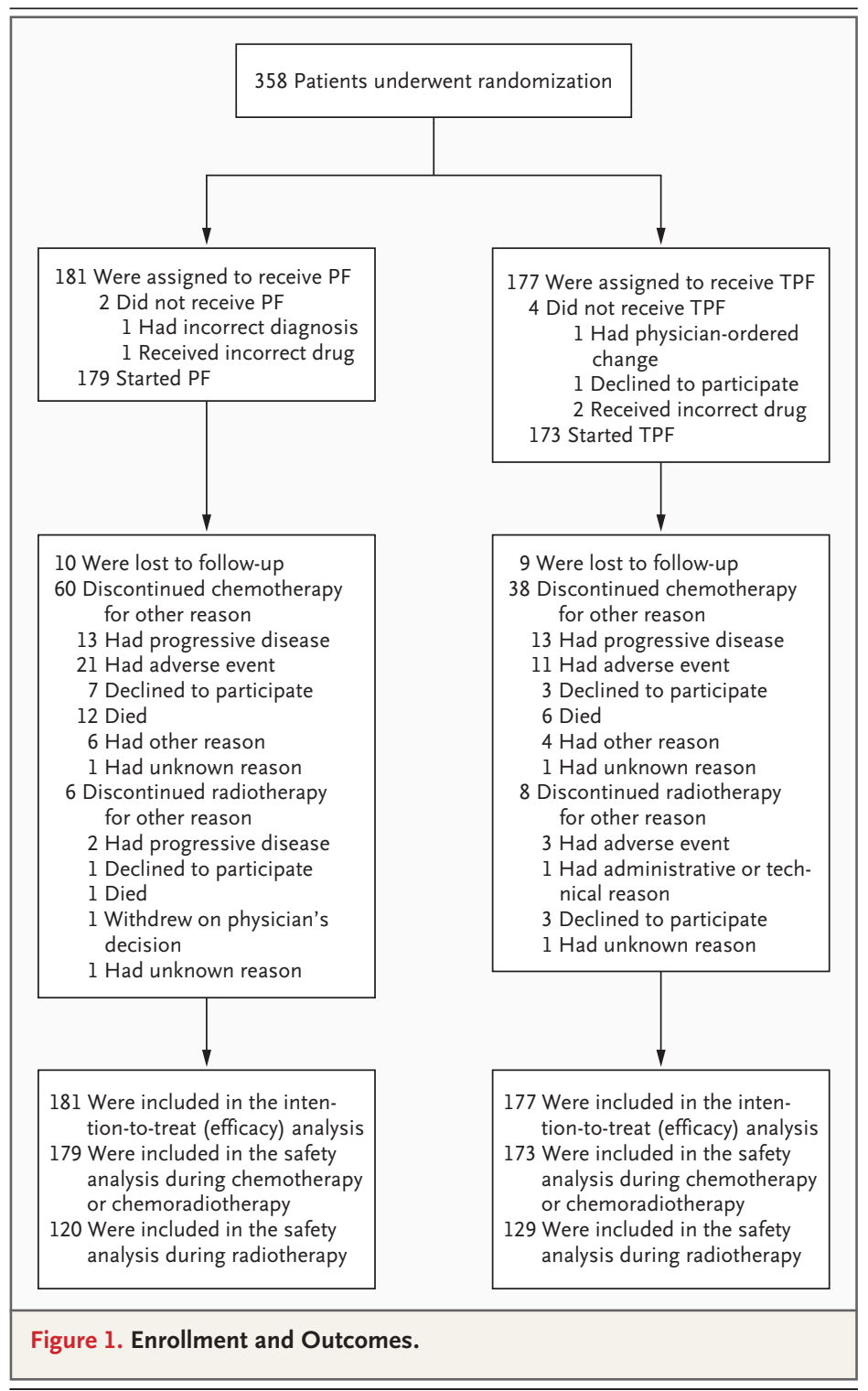

(5\%) and five in the PF group (3\%) discontinued the planned radiotherapy. Radiotherapy that was not defined in the protocol was administered to 27 of 44 patients in the TPF group (61\%) and 32 of 59 patients in the PF group (54\%) who had a recurrence while receiving induction chemotherapy. In total, 17 patients in the TPF group (10\%) and 27 patients in the PF group (15\%) did not receive radiotherapy $(\mathrm{P}=0.15)$.

\section{EFFICACY}

After a median follow-up of 32.5 months, a total of 273 patients had disease progression or had died (126 in the TPF group and 147 in the PF group). Treatment with TPF resulted in a relative reduction of $28 \%$ in the risk of disease progression or death, as compared with PF $(\mathrm{P}=0.007)$ (Fig. 2A) and significantly improved median progression-free survival, median overall survival, and time to treatment failure (Table 2).

The overall response rate in the TPF group was significantly higher than that in the PF group, both in the induction-chemotherapy phase and after locoregional therapy. Although the completeresponse rate was similar in the two groups in the induction-chemotherapy phase, it was significantly higher in the TPF group than in the PF group after locoregional therapy (33.3\% vs. $19.9 \%$, $\mathrm{P}=0.004$ ) (Table 2).

In a survival update performed on July 18 , 2005, at a median follow-up of 51.1 months, 268 patients had died (122 in the TPF group and 146 in the PF group) (Fig. 2B).

\section{PATTERN OF FIRST RELAPSE}

The total number of relapses was 101 in the TPF group (57.1\%) and 117 in the PF group (64.6\%). Most first relapses occurred at the locoregional disease site $(85.1 \%$ in the TPF group and $81.2 \%$ in the PF group). Distant relapses were observed in $12.9 \%$ of patients in the TPF group and in $10.3 \%$ in the PF group. Of the 25 patients with distant metastases, 5 also had locoregional relapse.

\section{SURGERY}

Elective neck dissections were performed before radiotherapy in seven patients in the TPF group and four patients in the PF group and after radiotherapy in nine patients in the TPF group and three patients in the PF group. Thus, a total of 16 patients in the TPF group and 7 patients in the PF group underwent neck dissection $(\mathrm{P}=0.05)$. After disease progression, 42 patients -27 in the TPF group and 15 in the PF group - underwent surgery $(\mathrm{P}=0.05)$. Thus, a total of 60 patients underwent surgery of any type during the study: 40 in the TPF group and 20 in the PF group $(\mathrm{P}=0.003)$.

A separate survey was performed among the 42 patients with progressive disease to determine whether resection had a curative or palliative intent. For 28 patients (20 in the TPF group and 8 in the PF group), the surgical intent was palliative, and for 10 patients ( 4 in the TPF group and 6 in 
the PF group), it was curative. For four patients, the intent of surgery was unknown. No significant difference was observed in the distribution between the two groups $(\mathrm{P}=0.22)$.

\section{ADVERSE EVENTS}

During chemotherapy, the most frequently observed severe nonhematologic adverse events were alopecia, stomatitis, infections, nausea, vomiting, anorexia, diarrhea, and hearing loss (Table 3). Apart from alopecia and infections, these adverse events were more frequent in the PF group than in the TPF group. Grade 3 or 4 neutropenia and leukopenia were more common in the TPF group, and severe thrombocytopenia and anemia were more common in the PF group. Febrile neutropenia occurred in $5.2 \%$ of patients in the TPF group and $2.8 \%$ in the PF group.

During radiotherapy, the most frequent treatment-related severe adverse events were stomatitis $23.7 \%$ in the TPF group and $20.7 \%$ in the PF group) and a combination of esophagitis, dysphagia, and odynophagia (13.9\% in the TPF group and $15.6 \%$ in the PF group).

Deaths associated with toxic effects occurred in 4 patients in the TPF group (2.3\%) and in 10 patients in the PF group (5.5\%) $(\mathrm{P}=0.17)$.

\section{DISCUSSION}

Our study showed that induction chemotherapy with TPF resulted in significant and clinical meaningful improvements in outcomes, as compared with $\mathrm{PF}$, in locoregionally advanced, unresectable squamous-cell carcinoma of the head and neck. Patients who were treated with TPF had a reduction of $28 \%$ in the risk of disease progression or death, as compared with those who received PF. They also had an extension of 2.8 months in median progression-free survival. This result was associated with significant improvements in overall survival, overall response rates, and time to treatment failure. Patients in the TPF group had a reduction of $27 \%$ in the risk of death, an improvement in median overall survival of 4.3 months, and an absolute increase in 3-year survival of 10.9\%.

Selection of the TPF regimen that was used in this phase 3 study was based on results of an earlier phase 1 and 2 study of four cycles of TPF used as induction chemotherapy in a similar pa-

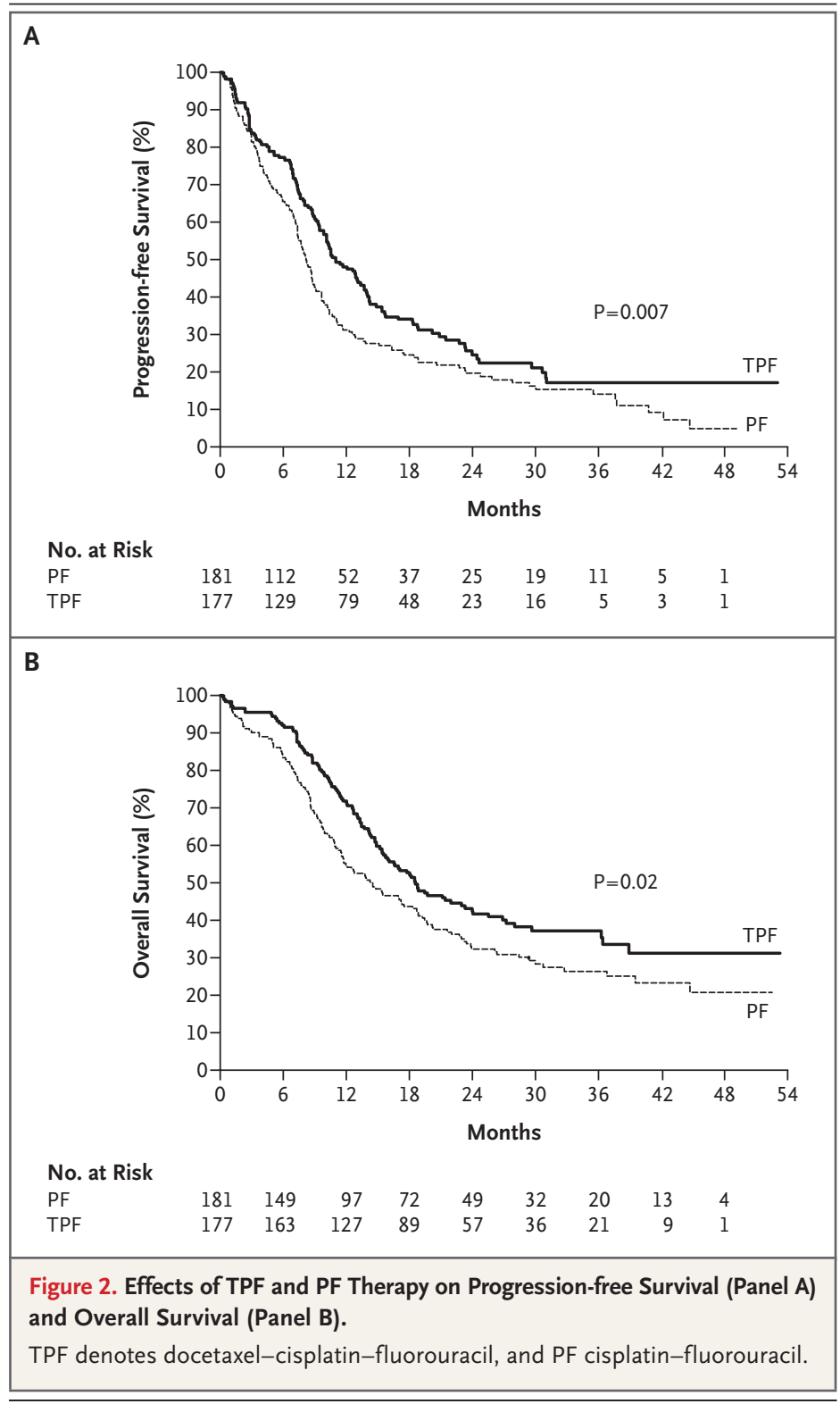

tient population. ${ }^{16}$ The median survival among the 48 patients treated in that study was 18.5 months; the rate of survival at 12,24 , and 36 months was $69 \%, 41 \%$, and $31 \%$, respectively. These data are remarkably similar to those in the TPF group in our phase 3 trial. However, our results in the TPF group are inferior to those of other phase 2 studies, which showed 2-year survival rates of 60 to $83 \%$ and overall response rates of 88 to $100 \%$; this difference may be due to the heterogeneity of the patient populations. ${ }^{17}$ 


\begin{tabular}{|c|c|c|c|c|}
\hline Variable & $\begin{array}{c}\mathrm{PF} \\
(\mathrm{N}=181)\end{array}$ & $\begin{array}{c}\text { TPF } \\
(\mathrm{N}=177)\end{array}$ & Hazard Ratio $(95 \% \mathrm{CI})$ & P Value \\
\hline \multicolumn{5}{|l|}{ Progression-free survival } \\
\hline Median duration - mo & 8.2 & 11.0 & $0.72(0.57-0.91)$ & $0.007 \dagger$ \\
\hline \multicolumn{5}{|l|}{ Rate - \% } \\
\hline At $l \mathrm{yr}$ & 31 & 48 & & \\
\hline At $2 \mathrm{yr}$ & 20 & 25 & & \\
\hline At $3 \mathrm{yr}$ & 14 & 17 & & \\
\hline \multicolumn{5}{|l|}{ Overall survival } \\
\hline Median duration - mo & 14.5 & 18.8 & $0.73(0.56-0.94)$ & $0.02 \dagger$ \\
\hline \multicolumn{5}{|l|}{ Rate - \% } \\
\hline At $1 \mathrm{yr}$ & 55 & 72 & & \\
\hline At $2 \mathrm{yr}$ & 32 & 43 & & \\
\hline At $3 \mathrm{yr}$ & 26 & 37 & & \\
\hline \multicolumn{5}{|l|}{ Response to chemotherapy } \\
\hline Overall — \% & 54 & 68 & & $0.006 \%$ \\
\hline Complete - no. (\%) & $12(6.6)$ & $15(8.5)$ & & \\
\hline Partial - no. (\%) & $85(47.0)$ & $105(59.3)$ & & \\
\hline \multicolumn{5}{|c|}{ Response to chemotherapy and radiotherapy } \\
\hline Overall - \% & 59 & 72 & & $0.006 \div$ \\
\hline Complete — no. (\%) & $36(19.9)$ & $59(33.3)$ & & 0.004 \\
\hline Partial — no. (\%) & $70(38.7)$ & $69(39.0)$ & & \\
\hline Duration of response - mo & 11.6 & 15.4 & $0.74(0.53-1.03)$ & $0.08 \int$ \\
\hline Time to treatment failure - mo & 7.8 & 10.5 & $0.70(0.55-0.89)$ & $0.003 \dagger$ \\
\hline
\end{tabular}

In our trial, survival was shorter than that reported in two other phase 3 trials of taxanecontaining induction chemotherapy regimens. ${ }^{18,19}$ The U.S. trial, a study by Posner et al. ${ }^{18}$ of concurrent chemotherapy and radiotherapy after induction chemotherapy (TAX 324), used a modified TPF regimen. The Spanish trial by Hitt et al. ${ }^{19}$ used PF plus paclitaxel (PPF). However, these trials included both patients with resectable disease and those with unresectable disease. In the study by Posner et al., reported in this issue of the Journal, the median survival was 71 months in the TPF group and 30 months in the PF group. The Spanish study showed a significant survival benefit only in patients with unresectable disease (median survival, 36 months in the PPF group and 26 months in the PF group). In contrast, in the study by Paccagnella et al.,, 7 the survival curve for patients with unresectable disease who were treated with $\mathrm{PF}$ followed by radiotherapy was identical to that for the PF group in our study.

As expected, TPF induced more leukopenia and neutropenia than PF but did not lead to more frequent infectious complications when patients received prophylactic antibiotics. Overall, TPF led to fewer treatment delays and was associated with fewer deaths from toxic effects (2.3\% in the TPF group and $5.5 \%$ in the PF group). PF was associated with more grade 3 or 
4 events of thrombocytopenia, nausea, vomiting, stomatitis, and hearing loss than was TPF. The improved tolerability of the triple regimen was also observed in the Spanish trial of PPF but was less evident in the TAX 324 study. The reduced doses of fluorouracil and cisplatin administered in our study probably reduced the toxicity.

Rates of locoregional control and survival improve more after accelerated or hyperfractionated radiotherapy than after conventionally fractionated radiotherapy. ${ }^{20,21}$ At the time our trial started, these radiotherapy standards had not been universally accepted within the EORTC's Head and Neck Cancer Group, so most of our patients received standard fractionation. However, even with these improvements, the outcomes for patients with unresectable disease remain dismal. Our study and the study by Posner et al. $^{18}$ raise two questions. First, is induction TPF followed by radiotherapy alone equivalent or superior to chemoradiotherapy? Second, do induction chemotherapy and chemoradiotherapy have complementary effects on overall control of disease? At least five ongoing randomized trials evaluating induction chemotherapy followed by chemoradiotherapy may provide the answer. ${ }^{22-24}$

In conclusion, our study showed that the addition of docetaxel to PF induction chemotherapy in patients with unresectable squamous-cell carcinoma of the head and neck improved survival and was better tolerated than the classic PF regimen.

Sponsored by Sanofi-Aventis and the National Cancer Institute.

Drs. Vermorken, Bernier, and Lefebvre report serving on paid advisory boards for Sanofi-Aventis; Dr. Preiss, having an equity interest in Bayer; and Dr. Rey, being an employee of SanofiAventis. No other potential conflict of interest relevant to this article was reported.
Table 3. Grade 3 or 4 Hematologic and Nonhematologic Adverse Events during Chemotherapy (Safety Population).*

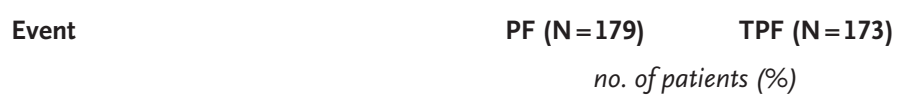

Hematologic'"

Neutropenia

$94(52.5)$

$133(76.9)$

Anemia

$23(12.8)$

$16(9.2)$

Thrombocytopenia

$32(17.9)$

$9(5.2)$

Leukopenia

$41(22.9)$

$72(41.6)$

Nonhematologic:

Alopecia

0

$20(11.6)$

Nausea

$12(6.7)$

$1(0.6)$

Stomatitis

20 (11.2)

8 (4.6)

Lethargy

$3(1.7)$

$5(2.9)$

Diarrhea

6 (3.4)

5 (2.9)

Vomiting

$8(4.5)$

$1(0.6)$

Neurotoxicity

$1(0.6)$

Anorexia

$6(3.4)$

Infection

$11(6.1)$

$12(6.9)$

Febrile neutropenia

5 (2.8)

$9(5.2)$

Weight loss

$1(0.6)$

0

Local toxic effect

$1(0.6)$

$1(0.6)$

Constipation

$1(0.6)$

0

Hearing loss

5 (2.8)

0

Esophagitis, dysphagia, or odynophagia

Gastrointestinal pain

0

*PF denotes cisplatin-fluorouracil, and TPF docetaxel-cisplatin-fluorouracil. $\dagger$ Hematologic toxic effects are listed regardless of whether there was a relationship with a study drug.

$\ddagger$ Nonhematologic toxic effects were related to treatment.

\section{REFERENCES}

1. Parkin DM, Bray F, Ferlay J, Pisani P. Global cancer statistics, 2002. CA Cancer J Clin 2005;55:74-108.

2. Seiwert TY, Cohen EEW. State-of-theart management of locally advanced head and neck cancer. BrJ Cancer 2005;92:13418.

3. Lefebvre J-L. Current clinical outcomes demand new treatment options for SCCHN. Ann Oncol 2005;16:Suppl 6:vi7-vi12.

4. Dimery IW, Hong WK. Overview of combined modality therapies for head and neck cancer. J Natl Cancer Inst 1993;85: 95-111.
5. Paccagnella A, Orlando A, Marchiori C, et al. Phase III trial of initial chemotherapy in stage III or IV head and neck cancers: a study by the Gruppo di Studio sui Tumori della Testa e del Collo. J Natl Cancer Inst 1994;86:265-72.

6. Adelstein DJ, Li Y, Adams G, et al. An intergroup phase III comparison of standard radiation therapy and two schedules of concurrent chemoradiotherapy in patients with unresectable squamous cell head and neck cancer. J Clin Oncol 2003; 21:92-8.

7. Zorat PL, Paccagnella A, Cavaniglia G, et al. Randomized phase III trial of neoadjuvant chemotherapy in head and neck cancer: 10-year follow-up. J Natl Cancer Inst 2004;96:1714-7.

8. Domenge C, Hill C, Lefebvre JL, et al. Randomized trial of neoadjuvant chemotherapy in oropharyngeal carcinoma. Br J Cancer 2000;83:1594-8.

9. Rooney M, Kish J, Jacobs J, et al. Improved complete response rate and survival in advanced head and neck cancer after three-course induction therapy with 120 hour 5-FU infusion and cisplatin. Cancer 1985;55:1123-8. 
10. Pignon JP, Bourhis J, Domenge C, Designé L. Chemotherapy added to locoregional treatment for head and neck squamous-cell carcinoma: three metaanalyses of updated individual data. Lancet 2000;355:949-55.

11. Schrijvers D, Vermorken JB. Role of taxoids in head and neck cancer. Oncologist 2000;5:199-208.

12. Pignon JP, Syz N, Posner M, et al. Adjusting for patient selection suggests the addition of docetaxel to 5-fluorouracilcisplatin induction therapy may offer survival benefit in squamous cell cancer of the head and neck. Anticancer Drugs 2004; 15:331-40.

13. Freedman LS, White SJ. On the use of Pocock and Simon's method for balancing treatment numbers over prognostic factors in the controlled clinical trial. Biometrics 1976;32:691-4.

14. Bernier J, Coens C, Remenar E, et al. Impact on quality of life (QoL) of the addition of docetaxel (T) to neoadjuvant cisplatin plus 5-fluorouracil treatment in patients with locally advanced unresectable squamous cell carcinoma of the head and neck. J Clin Oncol 2006;24:18S. abstract. 15. Slud EV, Byar DP, Green SB. A comparison of reflected versus test-based con- fidence intervals for the median survival time, based on censored data. Biometrics 1984;40:587-600.

16. Schrijvers D, Van Herpen C, Kerger J, et al. Docetaxel, cisplatin and 5-fluorouracil in patients with locally advanced unresectable head and neck cancer: a phase I-II feasibility study. Ann Oncol 2004;15: 638-45.

17. Posner MR. Paradigm shift in the treatment of head and neck cancer: the role of neoadjuvant chemotherapy. Oncologist 2005;10:Suppl 3:11-9.

18. Posner MR, Hershock DM, Blajman CR, et al. Cisplatin and fluorouracil alone or with docetaxel in head and neck cancer. N Engl J Med 2007;357:1705-15.

19. Hitt R, López A, Martínez-Trufero J, et al. Phase III study comparing cisplatin plus fluorouracil to paclitaxel, cisplatin, and fluorouracil induction chemotherapy followed by chemoradiotherapy in locally advanced head and neck cancer. J Clin Oncol 2005;23:8636-45. [Erratum, J Clin Oncol 2006;24:1015.]

20. Bourhis J, Overgaard J, Audry H, et al. Hyperfractionated or accelerated radiotherapy in head and neck cancer: a metaanalysis. Lancet 2006;368:843-54.

21. Budach W, Hehr T, Budach V, Belka C,
Dietz K. A meta-analysis of hyperfractionated and accelerated radiotherapy and combined chemotherapy and radiotherapy regimens in unresected locally advanced squamous cell carcinoma of the head and neck. BMC Cancer 2006;6:28.

22. Paccagnella A, Buffoli A, Koussis H, et al. Randomized phase II trial of concomitant CT/RT versus TPF followed by concomitant CT/RT in locally advanced squamous cell carcinoma of the head and neck (LASCCHN). J Clin Oncol 2006;24: 18S. abstract.

23. Hitt R, Grau J, Lopez-Pousa A, et al. Randomized phase II/III clinical trial of induction chemotherapy (ICT) with either cisplatin/5-fluorouracil (PF) or docetaxel/ cisplatin/5-fluorouracil (TPF) followed by chemoradiotherapy (CRT) vs. CRT alone for patients (pts) with unresectable locally advanced head and neck cancer (LAHNC). J Clin Oncol 2006;24:18S. abstract.

24. Adelstein DJ, LeBlanc M. Does induction chemotherapy have a role in the management of locoregionally advanced squamous cell head and neck cancer? J Clin Oncol 2006;24:2624-8.

Copyright $\odot 2007$ Massachusetts Medical Society. 